\title{
Equipe de saúde e o brincar da criança com queimaduras
}

\author{
Healthcare team and the play \\ of the child with burns
}

Adriano Valério dos Santos AZEVÊDO

\section{Resumo}

A pesquisa objetivou analisar as percepções da equipe de saúde sobre o brincar da criança hospitalizada com queimaduras. Participaram da amostra 30 profissionais de saúde da Unidade de Tratamento de Queimados. Utilizou-se um questionário, e a análise destacou que a equipe de saúde considera relevante o brincar no hospital devido às contribuições para a adesão ao tratamento, evolução clínica, adaptação e interação social da criança com queimaduras. Na perspectiva desses profissionais, o brincar por meio da função terapêutica permite que a criança hospitalizada desenvolva a criatividade, enfrente a dor, e utilize o manejo nas situações de estresse, o que possibilita auxiliar o processo de recuperação da saúde integral. Os dados positivos evidenciam a importância do brincar para a criança na situação de doença e hospitalização.

Unitermos: Criança hospitalizada; Queimaduras; Recreção.

\begin{abstract}
The aim of this study was to analyze the perceptions of the healthcare team regarding the play of children hospitalized with burns. A total of 30 healthcare professionals of the Burns Treatment Unit composed the sample. A questionnaire was used and the analysis detected that the healthcare team considered play important in the hospital due its contributions to treatment adherence, clinical evolution, adaptation, and social interaction of the child with burns. From of these professional's perspective, play as a therapeutic function allows the hospitalized child to develop creativity, cope with pain, and manage situations of stress, which represent a possibility to assist the recovery process of integral health. The positive data evidenced the importance of play for the child in situation of illness and hospitalization.
\end{abstract}

Uniterms: Child, hospitalized; Burns; Recreation.

As pesquisas na área da Psicologia Pediátrica destacam que, durante o processo de hospitalização, a criança apresenta alterações no humor, regressões no comportamento relacionadas às fases anteriores de desenvolvimento, dificuldades nas habilidades sociais e transtornos do sono que repercutem na vivência com o acompanhante, representado com frequência pela genitora, que compartilha essas situações e que desenvolve quadros de estresse (Chiattone, 2003; Hall et al., 2006; Lindquist, 1993; Pérez-Ramos, 2006). Percebe-se

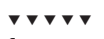

1 Universidade Federal de Santa Catarina, Centro de Filosofia e Ciências Humanas, Programa de Pós-Graduação em Psicologia. Campus Universitário, Trindade, 88040-500, Florianópolis, SC, Brasil. E-mail: <adrianoazevedopsi@yahoo.com>.

Apoio: Coordenação de Aperfeiçoamento de Pessoal de Nível Superior. 
que o adoecimento e a hospitalização provocam uma série de mudanças na vida da criança; segundo Lindquist (1993), as reações são decorrentes da separação da família, do surgimento da doença e da admissão no ambiente hospitalar.

O distanciamento do ambiente familiar e a necessidade de cumprir as regras da instituição hospitalar causam um impacto na esfera emocional das crianças hospitalizadas. O impacto representa um momento de choque, e a criança vivencia uma fase de sofrimento físico e psicológico. Pesquisadores indicam que a hospitalização pode representar um fator de risco para o desenvolvimento da criança quando o ambiente não proporciona condições adequadas para a expressão da linguagem e para o estabelecimento de relações sociais (Crepaldi, Rabuske \& Gabarra, 2006; Martins \& Paduan, 2010; Pérez-Ramos, 2006; Silva, 2006). Existe a necessidade de estruturar os ambientes hospitalares com recursos lúdicos para facilitar o acolhimento à criança, que, por muitas vezes, encontra-se assustada e agressiva. Isso pode ter uma relação com as sensações desagradáveis provenientes dos exames periódicos, assim como com os sentimentos de raiva atribuídos à família por não conseguir reduzir o tempo de hospitalização.

Os estudos destacam que as consequências provenientes do período de hospitalização ocasionam situações de crise entre a criança e sua família. Diante dos procedimentos invasivos, tais como curativos e cirurgias, a criança hospitalizada apresenta sensações de medo e abandono que provocam a perda da identidade (Chiattone, 2003; Crepaldi et al., 2006; Silva, 2006). Assim, o hospital representa um ambiente perigoso que provoca insegurança, já que a noção de doença e tratamento ocasiona insatisfação e potencializa a percepção de morte.

Nas instituições hospitalares, os indivíduos recebem o nome de pacientes, são identificados por números nas enfermarias, utilizam uniformes, e a assistência é realizada por meio de uma equipe multiprofissional. Além desses aspectos, existe a privação de liberdade, e a criança no hospital pode perceber essas ocorrências com sentimentos de hostilidade, o que gera comportamentos agressivos e conflitos com o acompanhante e equipe de saúde. De acordo com Dias, M.N.

58 Baptista e A.S.D. Baptista (2003), as crianças hospitalizadas com patologias distintas num período superior a cinco dias apresentam tendências para desenvolver transtornos psicológicos. Na visão dos autores, as reações psicológicas devem ser investigadas a partir de variáveis relevantes, tais como as experiências anteriores de internação, quadro clínico atual, vínculos familiares e o fator relacionado à idade. Chiattone (2003) destacou que as crianças nos dois primeiros anos de vida apresentam dificuldades para permanecer hospitalizadas devido às características do ambiente hospitalar, com paredes lisas, níveis variados de iluminação, pessoas estranhas e aparelhos específicos para a realização de exames. Importante destacar que existe um consenso entre os autores de que a hospitalização infantil integra múltiplos fatores, com repercussões para a criança, a família e a equipe de saúde.

Na hospitalização prolongada para o tratamento de queimaduras, a situação se torna complexa diante dos curativos utilizados para a recomposição da área lesionada e dos efeitos colaterais dos anestésicos. A queimadura é conceituada como uma lesão provocada por agentes externos que incluem fogo, choque elétrico, produtos químicos e demais fatores que danificam a pele e suas estruturas internas (Lima-Júnior, Novaes, Piccolo \& Serra, 2008). De acordo com esses autores, a avaliação da queimadura é realizada, conforme sua profundidade, a partir das classificações de 10, $2^{\circ}$ e $3^{\circ}$ graus, sendo as duas últimas consideradas mais graves; quanto à extensão da área atingida, atribui-se um escore quantitativo entre pequeno, médio e grande. A dor representa um fenômeno vivenciado pelos pacientes hospitalizados com queimaduras; dessa forma, o acompanhamento psicológico se torna fundamental para a criança e sua família. Stoddard et al. (2002) destacam que as intervenções psicológicas reduzem o nível de estresse da criança hospitalizada e permitem auxiliar o manejo das sensações dolorosas.

A assistência psicológica à criança hospitalizada deve ser realizada com o propósito de minimizar os efeitos nocivos provocados pelo adoecimento, buscando preservar os aspectos saudáveis do universo infantil por meio de recursos lúdicos. A American Academy of Pediatrics (AAP - 2006) recomenda a utilização das atividades lúdicas para as crianças hospitalizadas devido à importância para o desenvolvimento humano, o que permite facilitar o relacionamento da criança com o 
acompanhante e a reintegração no ambiente familiar e social.

Os autores da Psicologia do Desenvolvimento apresentam as contribuições do brincar para a expressão de afetos e resolução de conflitos, o que auxilia a espontaneidade da criança (Piaget, 1971), assim como a aprendizagem de habilidades sociais e a organização das experiências por meio dos jogos simbólicos que enfatizam a imaginação e os significados atribuídos às atitudes no mundo (Vigotski, 1998). Verificam-se os benefícios do brincar para as etapas de desenvolvimento da criança, o que permite integrar pontos relevantes relacionados ao desempenho de papéis sociais, a noção de indivíduo inserido nas relações interpessoais e o conhecimento acerca do mundo real e imaginário.

As atividades lúdicas representam o brincar em diversas modalidades, e, quando esse recurso é utilizado no hospital, trata-se de uma estratégia de humanização que valoriza as potencialidades das crianças, conforme foi apontado por Frota, Gurgel, Pinheiro, Martins e Tavares (2007). Importante ressaltar que essas ações nos ambientes de saúde consideram os aspectos globais de indivíduos e ampliam a noção de adoecimento, apresentando coerência com as propostas do Sistema Único de Saúde - SUS (2004). Esse programa do Ministério da Saúde focaliza a atenção, o cuidado e o respeito para os indivíduos que procuram os serviços de atendimento à saúde. No que se refere à hospitalização de crianças, o brincar humaniza o ambiente hospitalar e promove o resgate da concepção de vida.

Os estudos teóricos e empíricos (Chiattone, 2003; Silva, 2006; Zahr, 1998) evidenciam as contribuições do brincar para a integração da criança no ambiente hospitalar e para o enfrentamento das situações durante o período de tratamento (Carvalho \& Begnis, 2006; Lindquist, 1993; Motta \& Enumo, 2004). Existe um consenso entre os autores acerca dos benefícios das atividades lúdicas no contexto da hospitalização infantil; assim, a utilização do brincar representa uma ação de saúde focalizada na atenção integral às necessidades da criança.

Diante do trauma físico e psicológico ocasionado pela queimadura, a criança vivencia o período de hospitalização numa unidade fechada, com as limitações e regras da instituição, o que facilita o surgimento de sintomas de ansiedade relacionados à condição clínica e imagem corporal. Destaca-se que a ausência de atividades lúdicas na unidade de queimados pode propiciar o início de crises de pânico quando a criança é submetida ao tratamento da área queimada. Diante dessas repercussões psicológicas, os projetos lúdicos representam recursos facilitadores para a promoção da saúde, principalmente quando os profissionais das unidades de pediatria hospitalar priorizam a implantação dessa estratégia para auxiliar a recuperação da criança (Lindquist, 1993). Nesse contexto, verifica-se a necessidade de estudos científicos para investigar se as equipes de saúde atribuem importância para o brincar da criança hospitalizada.

Nas bases de dados BVS/PepSIC, Bireme/Lilacse Wiley/Interscience, no período de 1990-2010, relativamente às palavras-chave brincar no hospital e play and hospital, verificou-se a ausência de pesquisas com os profissionais de saúde da Unidade de Tratamento de Queimados (UTQ). Nessas consultas, foram identificados poucos estudos sobre as percepções das equipes de saúde acerca das atividades lúdicas das crianças hospitalizadas (Azevedo, Santos, Justino, Miranda \& Simpson, 2007; Cole, Diener, Wright \& Gaynard, 2001; Mello, Goulart, Ew, Moreira \& Sperb, 1999; Mitre \& Gomes, 2007; Picado, El-Khouri \& Streapco, 2007; Silva, 2006; Vieira \& Carneiro, 2006). Esses estudos indicam que os profissionais das unidades de pediatria geral e oncologia pediátrica consideram relevante o brincar no hospital devido aos benefícios para o desenvolvimento das habilidades adaptativas da criança. As pesquisas enfatizam, no entanto, apenas a área da oncologia infantil; assim, tratase de um ponto relevante investigar outros ambientes hospitalares e as percepções da equipe de saúde acerca do brincar. O baixo número de investigações científicas permite evidenciar que existe uma área de pesquisa a ser explorada, e, portanto a iniciativa de pesquisadores contribui para os avanços nesse campo de estudo.

Os estudos acerca da hospitalização infantil permitem identificar dados que orientam o desenvolvimento de práticas psicológicas e as ações de humanização nas instituições de saúde. Investigar as percepções da equipe de saúde sobre o brincar da criança hospitalizada com queimaduras possibilita a construção de formulações teóricas e o aprimoramento de pesquisas na área da Psicologia Pediátrica. O termo percepções se refere a um conjunto de ideias acerca de um 
determinado fenômeno, assim como as construções cognitivas que possibilitam a formação e definição de conceitos. Considerando-se a necessidade de produções científicas sobre a referida temática, justifica-se a relevância da presente pesquisa. Baseando-se na perspectiva da humanização nas práticas de saúde, esta pesquisa objetivou analisar as percepções da equipe de saúde sobre o brincar da criança hospitalizada com queimaduras.

\section{Método}

Este estudo descritivo foi realizado com a equipe de saúde da UTQ de um hospital na Região Nordeste do Brasil. Optou-se pela UTQ a partir do seguinte critério: 1) representa um setor fechado com uma dinâmica diferenciada de outros ambientes da instituição hospitalar devido ao período prolongado para o tratamento de queimaduras; 2) a unidade hospitalar é considerada referência para o tratamento de pacientes queimados e 3) a UTQ integra 40 profissionais de saúde de diversas áreas que trabalham em turnos distintos. É importante ressaltar que esses profissionais foram convidados para participação na pesquisa.

\section{Participantes}

Participaram da pesquisa 30 integrantes da equipe de saúde da UTQ: três anestesiologistas, três cirurgiões plásticos, dois pediatras, dois fisioterapeutas, dois enfermeiros, um clínico geral, um psicólogo, oito técnicos de enfermagem e oito auxiliares de enfermagem.

\section{Instrumento e procedimentos}

Foi construído um questionário com perguntas fechadas e abertas acerca das percepções quanto ao brincar da criança que está hospitalizada com queimaduras, considerando-se que as atividades lúdicas estavam sendo desenvolvidas na unidade hospitalar. Nas questões fechadas, procurou-se verificar o grau de importância atribuído ao brincar da criança no hospital, a utilidade do recurso lúdico no ambiente hospitalar e a opinião sobre a continuidade das atividades lúdicas na UTQ, solicitando-se justificativas para as respostas que 60 foram destacadas. Nas questões abertas, foram apre- sentadas perguntas sobre as percepções acerca do brincar no hospital e as possíveis contribuições das atividades lúdicas para a criança na UTQ.

Em uma reunião coletiva, os profissionais de saúde foram convidados para responder ao questionário na UTQ. OTermo de Consentimento Livre e Esclarecido foi anexado ao instrumento de pesquisa. O estudo foi aprovado pelo comitê de ética em pesquisa com seres humanos da instituição hospitalar.

Utilizou-se a análise de conteúdo de acordo com os princípios de Bardin (1979) a partir dos seguintes procedimentos: leitura integral das respostas, identificação de palavras e frases, destacando-se a ocorrência e frequência no texto. Em seguida, foram estabelecidas as unidades de codificação constituídas de palavras com significados semelhantes e que representam o conteúdo do material analisado, e, na etapa posterior, ocorreu o agrupamento dos dados com a formação de categorias temáticas. Destaca-se que dois juízes analisaram os dados, e o cálculo de concordância indicou o percentual de $75 \%$, o que garante a fidedignidade dos dados da pesquisa, considerando-se suficiente o referencial igual ou superior a 70\%, conforme destaca Fagundes (1999).

Procurou-se apresentar a frequência e a porcentagem referentes às temáticas que foram analisadas, assim como os relatos dos participantes para facilitar a compreensão dos dados. Foram definidos os elementos centrais que integram as temáticas, buscando-se compreender as especificidades do fenômeno (brincar no hospital) de forma específica para o grupo pesquisado. Os dados representam um modelo teórico sobre a complexidade do fenômeno. Ao definir as unidades que integram o brincar no hospital, pode-se compreender as inter-relações deste objeto de estudo.

\section{Resultados}

Todos os profissionais de saúde que participaram da pesquisa consideram importante o desenvolvimento do brincar da criança hospitalizada na UTQ e aprovam a continuidade das atividades lúdicas. Os relatos de dois profissionais relacionam o brincar a uma estratégia de humanização ("Importantíssimo para humanização do tratamento"; "No que concerne ao tratamento da criança internada e seus familiares, serão projetos antes de tudo humanísticos"). O ambiente 
hospitalar foi considerado por todos os integrantes da equipe de saúde um espaço de grande utilidade para proporcionar atividades lúdicas. Os relatos sobre o brincar no hospital indicam a importância desse recurso para o tratamento e o processo de recuperação da criança ("Desenvolve e ajuda bastante na recuperação da criança queimada"; "É muito importante para a criança no tratamento hospitalar'). Uma proporção menor de participantes $(n=2)$ sugeriu manter os brinquedos higienizados para evitar infecções nas crianças, assim como a organização dos brinquedos em um local específico na unidade hospitalar.

A Tabela 1 apresenta as principais respostas da equipe de saúde sobre as contribuições do brincar para a criança hospitalizada com queimaduras. A participação da criança nos procedimentos do setor representou a resposta mais citada. Em seguida, foram identificadas respostas sobre o enfrentamento da dor ("A dor que é aliviada através das atividades recreativas sem necessidade de analgesias"), efeito terapêutico ("Ajuda muito no bem-estar da criança hospitalizada"), habilidades adaptativas ("Promove melhor adaptação ao ambiente, permitindo com maior facilidade as condutas ou atémesmo complementando as condutas"), interação com outras pessoas ("A socialização e aproximaçãojunto dos profissionais"). Outro pequeno percentual de respostas destacou a redução da ansiedade e a promoção da saúde integral.

A análise de dados permitiu destacar que o brincar no contexto da hospitalização infantil apresenta repercussões para uma série de fatores. A Figura 1 re- presenta um modelo teórico que permite conceituar o fenômeno do brincar no hospital na unidade de queimados.

O brincar no hospital: (1) possibilita a adesão da criança ao tratamento e a inserção da criança nos procedimentos do setor relacionados aos curativos e às cirurgias; (2) facilita o enfrentamento da dor: os momentos destinados para o brincar permitem minimizar os níveis de dor e, em alguns casos, existe a ausência de queixas dolorosas nas áreas do corpo; (3) contribui para a função terapêutica: integra as sensações de relaxamento e bem-estar psíquico; (4) desenvolve a adaptação: a criança começa a cumprir os horários estabelecidos para a alimentação e sono, demonstrando receptividade às intervenções realizadas pela equipe de saúde; (5) contribui para a interação social: há o desenvolvimento de relações sociais entre a criança e os integrantes da equipe de saúde e os familiares; (6) favorece o manejo nas situações de estresse: o brincar permite que a criança utilize o manejo nas situações que envolvem curativos e fisioterapia, o que possibilita que ela entenda a importância dos procedimentos técnicos, o que facilita a atuação do profissional de saúde; (7) promove a evolução clínica: melhora significativa no quadro clínico relativo à queimadura; (8) desenvolve a criatividade: a criança manipula os brinquedos e inicia a construção de desenhos por meio de formas criativas; (9) contribui para a recuperação da saúde integral: efeitos positivos nos aspectos fisiológicos e psicológicos; e (10) reduz os níveis de ansiedade: as sensações de perigo são minimizadas, assim como o choro e as queixas acerca das

Tabela 1

Frequência e porcentagem das contribuições do brincar no hospital para a criança com queimaduras na percepção da equipe de saúde. Aracaju (SE), 2008

\begin{tabular}{lcc}
\hline Temas & Frequência $(n)$ & Percentual \\
\hline Participação nos procedimentos do setor & 28 & 15,9 \\
Enfrentamento da dor & 25 & 14,2 \\
Efeito terapêutico & 20 & 11,3 \\
Habilidades adaptativas & 20 & 11,3 \\
Interação com as pessoas & 20 & 11,3 \\
Manejo do estresse & 20 & 11,3 \\
Evolução no quadro clínico & 15 & 8,5 \\
Potencial criativo & 10 & 5,6 \\
Saúde integral & 10 & 5,6 \\
Minimiza a ansiedade & 8 & 4,5 \\
\hline Total & 176 & 100,0 \\
\hline
\end{tabular}




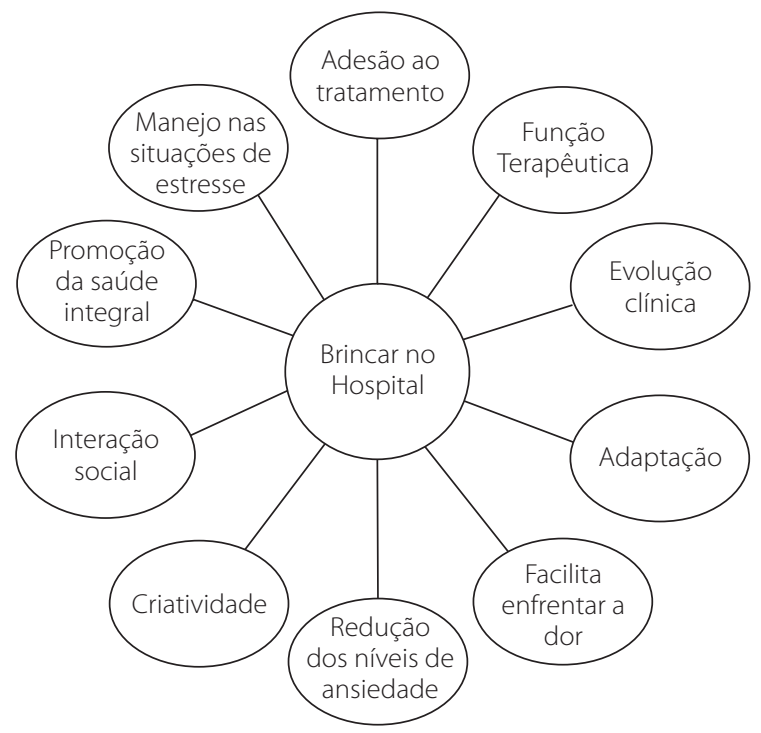

Figura 1. Modelo teórico sobre o brincar da criança hospitalizada com queimaduras.

intervenções da equipe de saúde. Os dados permitem compreender que o brincar no hospital representa um recurso com múltiplas funções que contribui para a vivência da criança na unidade de queimados.

\section{Discussão}

O principal interesse desta pesquisa foi analisar as percepções da equipe de saúde sobre o brincar da criança hospitalizada com queimaduras. Os dados positivos apresentam contribuições teóricas devido à relevância das atividades lúdicas na UTQ. Na literatura consultada, foi possível identificar os estudos científicos que enfatizam os efeitos do brincar no hospital.

No que se refere à importância atribuída pela equipe de saúde ao brincar da criança na unidade de queimados para auxiliar no tratamento e no processo de recuperação, os dados apresentam congruência com as pesquisas que foram realizadas com outros profissionais de saúde no contexto hospitalar (Azevedo et al., 2007; Cole et al., 2001; Mello et al., 1999; Picado et al., 2007; Silva, 2006; Vieira \& Carneiro, 2006). Esses dados representam um aspecto positivo para a construção de projetos lúdicos para as crianças hospitalizadas, considerando que a receptividade dos profissionais de saúde

62 possibilita desenvolver ações integradas.
O primeiro ponto destacado pela equipe de saúde foi a adesão da criança ao tratamento. Em psicologia pediátrica, o brincar é considerado o principal recurso para integrar a criança hospitalizada aos procedimentos necessários para a recuperação. Verificou-se que as pesquisas destacam a importância da preparação psicológica da criança para os procedimentos técnicos da unidade hospitalar por meio de informações educativas e atividades lúdicas (Chiattone, 2003; Silva, 2006; Zahr, 1998). A partir do brincar no hospital, a criança desenvolve a colaboração com os profissionais de saúde, o que representa um ponto fundamental durante o período de hospitalização.

A contribuição do brincar no hospital para a adaptação da criança foi destacada pela equipe de saúde. As pesquisas evidenciam que a principal função do brincar no hospital é promover um ambiente seguro para desenvolver a adaptação na criança (AAP, 2006; Carvalho \& Begnis, 2006). Dessa forma, o desenvolvimento das habilidades adaptativas permite facilitar a inserção da criança no hospital e a construção das redes de apoio.

Os estudos científicos indicam que o brincar no hospital auxilia a criança na utilização de estratégias cognitivas e comportamentais com o objetivo de reduzir os níveis de ansiedade (Lindquist, 1993; Oliveira, Dias \& Roazzi, 2003; Zahr, 1998). Percebe-se que o brincar auxilia a regulação emocional das crianças hospitalizadas, facilitando o manejo nas situações de estresse; assim, os dados da presente pesquisa apresentam congruência com os estudos da literatura. Os benefícios do brincar para o desenvolvimento cognitivo, afetivo e social da criança e a importância dos projetos lúdicos no ambiente hospitalar para a promoção da qualidade de vida foram destacados por diversos autores (Domingues \& Motti, 2008; Hendon \& Bohon, 2008; Pérez-Ramos, 2006; Silva, 2006). Nota-se que as atividades lúdicas valorizam a dimensão humana, o estabelecimento da comunicação e a construção de habilidades sociais.

Os participantes da presente pesquisa atribuíram importância para o brincar da criança hospitalizada devido aos benefícios para a função terapêutica, criatividade e recuperação da saúde. Os autores destacam os efeitos terapêuticos do brincar no hospital, considerando relevante o desenvolvimento de aspectos criativos da 
criança e as repercussões positivas nos fatores fisiológicos e psicológicos (Chiattone, 2003; Pérez-Ramos, 2006). No estudo de Picado et al. (2007), as intervenções musicoterapêuticas com as crianças hospitalizadas foram consideradas pelos profissionais de saúde recursos benéficos para a saúde física e mental. Os estudos (Ginsburg, Committe on Communications \& Comitteee on Psychosocial Aspects of Child and Family Health, 2007; Pérez-Ramos, 2010; Soares \& Zamberlan, 2001) evidenciam a importância do brincar para a saúde da criança e o desenvolvimento e manu-tenção de vínculos afetivos com a família. Essas pes-quisas destacam as contribuições do brincar no hospital para a promoção da saúde integral, assim percebe-se que as intervenções lúdicas representam recursos potencializadores para o desenvolvimento saudável da criança.

Neste estudo, o brincar foi considerado um recurso importante para o estabelecimento da interação social e o enfrentamento da dor provocada pelas queimaduras, aspectos fundamentais para o paciente hospitalizado na unidade de queimados. Dados positivos relacionados à capacidade de comunicação das crianças por meio do brincar no hospital foram evidenciados em outras pesquisas (AAP, 2006; Chiattone, 2003; Frota et al., 2007; Motta \& Enumo, 2004; Silva, 2006) e mostraram que o desenvolvimento das relações interpessoais facilita a inserção da criança no ambiente hospitalar e o diálogo com o acompanhante e a equipe de saúde.

Em virtude das queimaduras, a criança vivencia sensações dolorosas nas áreas lesionadas, e o enfrentamento de forma adaptativa contribui para o manejo adequado da dor. Pesquisadores destacam a importância das atividades lúdicas para as crianças hospitalizadas que são submetidas aos tratamentos dolorosos no corpo (Stoddard et al., 2002). As queimaduras ocasionam nas crianças hospitalizadas mudanças fisiológicas e psicológicas que têm repercussões, durante o tratamento, nos seguintes aspectos: relacionamento com a equipe de saúde e a família, dificuldades para reconhecer a importância dos procedimentos técnicos e problemas relacionados à autoimagem. Dessa forma, a promoção do brincar na unidade de queimados deve ser considerada relevante para facilitar o processo de recuperação da criança e sua adaptação ao tratamento de queimaduras. Destaca-se a percepção da equipe de saúde sobre a temática relacionada ao enfrentamento da dor por meio do brincar como um novo dado para ser contextualizado nas pesquisas, o que pode facilitar o manejo da criança nos procedimentos invasivos.

Mitre e Gomes (2007) analisaram as possibilidades e os limites acerca do brincar no hospital na visão da equipe de saúde. Verificou-se que existem dificuldades relacionadas à falta de reconhecimento da instituição de saúde e de outros profissionais acerca da utilização do brincar. No estudo de Martins e Paduan (2010), a equipe de saúde considerou relevante o brincar no hospital, mas os profissionais não se percebem mediadores para o desenvolvimento psicossocial da criança hospitalizada. Um ponto fundamental refere-se à necessidade de formação e integração da equipe de saúde para compreender as funções do brincar.

Na pesquisa realizada por Silva (2006), a equipe de saúde destacou que as atividades lúdicas auxiliam a recuperação da criança hospitalizada e permitem reduzir a apatia, promovendo momentos de satisfação e mudanças positivas no humor. Os profissionais de saúde que participaram do estudo de Vieira e Carneiro (2006) reconhecem a importância do brincar no hospital para a criança, e os aspectos positivos incluíram as sensações prazerosas, a redução de tensões e o estabelecimento da comunicação. Essas pesquisas apontam a relevância do brincar no hospital e os dados estão coerentes com o presente estudo.

Lindquist (1993) destacou a importância de a equipe de saúde reconhecer a função do lúdico no hospital para ampliar as possibilidades de tratamento da criança hospitalizada. Dessa forma, os fatores fisiológicos e psicológicos resultantes da doença representam o foco para o desenvolvimento de intervenções, em contraposição ao modelo reducionista que prioriza o funcionamento orgânico. Esse novo olhar para as repercussões psicológicas que surgem a partir da situação de adoecimento permite destacar que a criança precisa do apoio da família e da equipe de saúde para enfrentar as vivências do período de hospitalização.

Um ponto relevante refere-se à percepção da equipe de saúde da UTQ acerca dos benefícios do brincar para a vivência da criança hospitalizada. Mostra-se pertinente a construção de um ambiente acolhedor que 
priorize a singularidade do indivíduo; dessa forma, o comprometimento dos profissionais de saúde com essa tarefa contribui para as ações efetivas.

\section{Considerações Finais}

Na visão da equipe de saúde da UTQ, o brincar apresenta uma série de contribuições para a criança hospitalizada com queimaduras. Os dados obtidos apresentam congruência com a literatura consultada, e a integração das temáticas possibilitou compreender as características do brincar no hospital. Os próximos estudos podem contextualizar esses dados para ampliar as estratégias de investigação em psicologia pediátrica.

Importante ressaltar as limitações do estudo que estão relacionadas ao instrumento de pesquisa. O questionário foi construído com poucas perguntas devido ao tempo restrito dos profissionais de saúde para participar de pesquisa. Assim, acredita-se que a utilização da entrevista nos próximos estudos representa um recurso relevante. Existe a necessidade de se investigarem as iniciativas que são desenvolvidas pelas instituições hospitalares para a promoção do brincar, e novos estudos podem ser realizados para verificar o processo de construção e execução de projetos lúdicos no ambiente hospitalar.

Os profissionais de saúde da UTQ destacam que a adesão ao tratamento, evolução clínica, função terapêutica, criatividade, adaptação, interação social, enfrentamento da dor, redução da ansiedade, manejo nas situações de estresse e promoção da saúde integral representam as principais contribuições do brincar no hospital para a criança com queimaduras.

Diante dos dados obtidos nesta pesquisa, torna-se necessário promover ações coletivas de saúde para minimizar o impacto emocional da criança no ambiente hospitalar; dessa forma, a utilização de atividades lúdicas representa uma alternativa eficaz.

\section{Referências}

American Academy of Pediatrics. (2006). Child life council and committee on hospital care child life services. Pediatrics, 118(4), 1757-1763.

Azevedo, D. M., Santos, J. J. S., Justino, M. A. R., Miranda, F. A. N., \& Simpson, C. A. (2007). O brincar como instrumento terapêutico na visão da equipe de saúde. Ciência Cuidado e Saúde, 6(3), 335-341.

Bardin, L. (1979). Análise de conteúdo. Lisboa: Edições 70.

Carvalho, A. M., \& Begnis, J. G. (2006). Brincar em unidades de atendimento pediátrico: aplicações e perspectivas. Psicologia em Estudo, 11(1), 109-117.

Chiattone, H. B. C. (2003). A criança e a hospitalização. In V. A. A. Camon (Org.), A psicologia no hospital (2a ed., pp.23-99). São Paulo: Pioneira Thomson Learning.

Cole, W., Diener, M., Wright, C., \& Gaynard, L. (2001). Health care professional's perceptions of child life specialists. Children's Health Care, 30(1), 1-15.

Crepaldi, M. A., Rabuske, M. M., \& Gabarra, L. M. (2006). Modalidades de atuação do psicólogo em psicologia pediátrica. In M. A. Crepaldi, M. B. M. Linhares \& G. B. Perosa (Orgs.), Temas em psicologia pediátrica (pp.13-55). São Paulo: Casa do Psicólogo.

Dias, R. R., Baptista, M. N., \& Baptista, A. S. D. (2003). Enfermaria de pediatria: avaliação e intervenção psicológica. In R. R. Dias, M. N. Baptista \& A. S. D. Baptista (Orgs.), Psicologia hospitalar: teoria, aplicações e casos clínicos (pp.53-73). Rio de Janeiro: Guanabara.

Domingues, A. F., \& Motti, T. F. G. (2008). O brincar e as habilidades sociais da criança com deficiência auditiva e mãe ouvinte. Estudos de Psicologia (Campinas), 25(1), 37-44. doi: 10.1590-50103-166X200800010004.

Fagundes, A. J. S. M. (1999). Descrição, definição e registro do comportamento. São Paulo: Edicon.

Frota, M. A., Gurgel, A. A., Pinheiro, M. C. D., Martins, M. C., \& Tavares, T. A. N. R. (2007). O lúdico como instrumento facilitador na humanização do cuidado de crianças hospitalizadas. Cogitare Enfermagem, 12(1), 69-75.

Ginsburg, K. R., Committe on Communications, \& Comitteee on Psychosocial Aspects of Child and Family Health. (2007). The importance of play in promoting healthy child development and maintaining strong parent-child bonds. Pediatrics, 119(1), 182-191.

Hall, E., Saxe, G., Stoddard, F., Kaplow, J., Koenen, K., Chawla, N., et al. (2006). Posttraumatic stress symptoms in parents of children with acute burns. Journal of Pediatric Psychology, 31(4), 403-412.

Hendon, C., \& Bohon, L. M. (2008). Hospitalized children's mood differences during play and music therapy. Child: Care, Health and Development, 34(2), 141-144.

Lima-Júnior, E. M., Novaes, F. N., Piccolo, N. S., \& Serra, M. C. V. F. (2008). Tratado de queimaduras no paciente agudo (2a ed.). São Paulo: Atheneu.

Lindquist, I. (1993). A criança no hospital:terapiapelo brinquedo. São Paulo: Scritta.

Martins, S. T. F., \& Paduan, V. C. (2010). A equipe de saúde como mediadora no desenvolvimento psicossocial da criança hospitalizada. Psicologia em Estudo, 15(1), 45-54.

Mello, C. O., Goulart, C. M. T., Ew, R. A., Moreira, A. M., \& Sperb, T. M. (1999). Brincar no hospital: assunto para discutir e praticar. Psicologia: Teoria e Pesquisa, 15(1), 65-74. 
Mitre, R. M. A., \& Gomes, R. (2007). A perspectiva dos profissionais de saúde sobre a promoção do brincar em hospitais. Ciência \& Saúde Coletiva, 12(5), 1277-1284.

Motta, A. B., \& Enumo, S. R. F. (2004). Câncer infantil: uma proposta de avaliação das estratégias de enfrentamento da hospitalização. Estudos de Psicologia (Campinas), 21(3), 193-202. doi: $10.1590 .50103-166 \times 200400300004$.

Oliveira, S. S. G., Dias, M. G. B. B., \& Roazzi, A. (2003). O lúdico e suas implicações nas estratégias de regulação emocional em crianças hospitalizadas. Psicologia: Reflexão e Crítica, 16(1), 1-13.

Pérez-Ramos, A. M. Q. (2006). O ambiente na vida da criança hospitalizada. In E. Bomtempo, E. G. Antunha \& V. B. Oliveira (Orgs.), Brincando na escola, no hospital, na rua (pp.111-126). Rio de Janeiro: Wak.

Pérez-Ramos, A. M. Q. (2010). Progressos científicos na qualidade de vida das crianças: o lúdico como estratégia preventiva. In A. M. Q. Pérez-Ramos \& V. B. Oliveira (Orgs.), Brincar é saúde: o lúdico como estratégia preventiva (pp.23-40). Rio de Janeiro: Wak.

Piaget, J. (1971). A formação do símbolo na criança. Rio de Janeiro:Zahar.

Picado, S. B. R., El-Khouri, R. N., \& Streapco, P. T. (2007). Humanização hospitalar infantil: intervenções musicoterapêuticas no Centro Clínico Electra Bonini. Pediatria, 29(2), 99-108.

Silva, S. M. M. (2006). Atividades lúdicas e crianças hospitalizadas por câncer: o olhar dos profissionais e das voluntárias. In E. Bomtempo, E. G. Antunha \& V. B. Oliveira (Orgs.), Brincando na escola, no hospital, na rua (pp.127141). Rio de Janeiro: Wak.

Sistema Único de Saúde. (2004). Política nacional de humanização: a humanização como eixo norteador das práticas de atenção e gestão em todas as instâncias do SUS. Brasília: Ministério da Saúde.

Soares, M. R. Z., \& Zamberlan, M. A. T. (2001). A inclusão do brincar na hospitalização infantil. Estudos de Psicologia (Campinas), 18(2), 64-69.

Stoddard, F. J., Sheridan, R. L., Saxe, G. N., King, B. S., King, B. H., Chedekel, D. S., et al. (2002). Treatment of pain in acutely burned children. Journal of Burn Care \& Rehabilitation, 23(2), 135-156.

Vieira, T., \& Carneiro, M. (2006). O brincar na sala de espera de um ambulatório pediátrico: possíveis significados. In E. Bomtempo, E. G. Antunha \& V. B. Oliveira (Orgs.), Brincando na escola, no hospital, na rua (pp.75-110). Rio de Janeiro: Wak.

Vigotski, L. S. (1998). A formação social da mente. São Paulo: Martins.

Zahr, L. K. (1998). Therapeutic play for hospitalized preschoolers in Lebanon. Pediatric Nursing, 24(5), 449-454.

Recebido em: 10/1/2011

Versão final em: 11/7/2012

Aprovado em: 23/8/2012 
